\title{
The utility of hand-held mobile spirometer technology in a resource-constrained setting
}

\author{
E du Plessis, ${ }^{1} \mathrm{MB}$ ChB; F Swart, ${ }^{1}$ NDip Clinical Technology (Pulmonology \& Critical Care); \\ D Maree, ${ }^{1}$ NDip Clinical Technology (Pulmonology \& Critical Care); J Heydenreich, ${ }^{1}$ NDip Clinical Technology (Pulmonology \& Critical Care); \\ J van Heerden, ${ }^{1}$ NDip Clinical Technology (Pulmonology); T M Esterhuizen, ${ }^{2}$ MSc; E M Irusen, ${ }^{1}$ MB ChB, FCP (SA), FCCP, PhD; \\ C F N Koegelenberg, ${ }^{1}$ MB ChB, MMed (Int), FCP (SA), FRCP, Cert Pulm (SA), PhD \\ ${ }^{1}$ Division of Pulmonology, Department of Medicine, Faculty of Medicine and Health Sciences, Stellenbosch University and Tygerberg Academic \\ Hospital, Cape Town, South Africa \\ ${ }^{2}$ Division of Epidemiology and Biostatistics, Department of Global Health, Tygerberg Hospital and Faculty of Medicine and Health Sciences, \\ Stellenbosch University, Cape Town, South Africa
}

Corresponding author: E du Plessis (elanadup@icloud.com)

Background. Mobile phone-linked spirometry technology has been designed specifically for evaluating lung function at primary care level. The Air-Smart Spirometer is the first mobile spirometer accepted in Europe for the screening of patients with chronic respiratory diseases. Objectives. To prospectively assess the accuracy of the device in measuring forced expiratory volume in the first second $\left(\mathrm{FEV}_{1}\right)$ and forced vital capacity (FVC) in a South African population, and to investigate the ability of the device to detect obstructive ventilatory impairment. Methods. A total of 200 participants were randomly assigned to perform spirometry with either the mobile spirometer connected to a smartphone or the desktop spirometer first, followed by the other. The $\mathrm{FEV}_{1} / \mathrm{FVC}$ ratio as well as the absolute $\mathrm{FEV}_{1}$ and $\mathrm{FVC}$ measurements were compared, using each participant as their own control. A Pearson correlation and Bland-Altman analysis were performed to measure the agreement between the two devices. We defined obstructive ventilatory impairment as $\mathrm{FEV}_{1} / \mathrm{FVC}<0.7$ measured by desktop spirometry in order to calculate the sensitivity, specificity, positive predictive value (PPV) and negative predictive value (NPV) of the Air-Smart Spirometer. Results. There was a strong correlation between the absolute $\mathrm{FEV}_{1}$ and $\mathrm{FVC}$ values and $\mathrm{FEV}_{1} / \mathrm{FVC}$ ratio measured with the mobile AirSmart Spirometer and more conventional pulmonary function testing, with $r=0.951, r=0.955$ and $r=0.898$, respectively. The Air-Smart Spirometer had a sensitivity of $97.6 \%$, specificity of $74.4 \%$, PPV of $73.0 \%$ and NPV of $97.8 \%$ for obstructive ventilatory impairment. Conclusions. The mobile Air-Smart Spirometer compared well with conventional spirometry, making it an attractive and potentially affordable tool for screening purposes in a primary care setting. Moreover, it had a high sensitivity and NPV for obstructive ventilatory impairment.

S Afr Med J 2019;109(4):219-222. DOI:10.7196/SAMJ.2019.v109i4.13845

Many southern African countries have undergone rapid demographic changes over the past decades, mainly attributable to urbanisation. ${ }^{[1]}$ This demographic shift has led to a rise in noncommunicable diseases against the background of a high prevalence of HIV infection and an alarming incidence of tuberculosis. ${ }^{[2]}$ The concept of 'colliding epidemics' is of particular importance in South Africa (SA), where we are faced with several major epidemics including HIV, tuberculosis and chronic obstructive pulmonary disease (COPD), both from cigarette smoking and from pollution, all of which may impact significantly on respiratory health. ${ }^{[3,4]}$

Lack of access to appropriate surveillance tools such as spirometry poses a major barrier to diagnosis and appropriate treatment in patients with chronic respiratory ailments such as COPD and asthma in poorly resourced countries. Despite the high prevalence in the general population, obstructive airway diseases continue to be underdiagnosed. ${ }^{[5,6]}$

Formal spirometry forms an integral part of the diagnosis and management of patients with asthma and COPD and relies on a competent operator, accurate equipment, standardised operating procedures, quality control and patient co-operation as outlined in the South African guideline for office spirometry. ${ }^{[7]}$

Mobile phone-linked spirometry technology has been designed specifically for evaluating lung function at primary care level, allowing patients access to potential diagnosis, monitoring and treatment in ways that are currently not available in these settings.

Several mobile spirometers have been developed over the years, most of which have several limitations, including not displaying a flow/volume loop. ${ }^{[8-12]}$ The Air-Smart Spirometer with a loop display is the first mobile spirometer accepted in Europe for the cost-effective screening of patients with chronic respiratory diseases. ${ }^{[13]}$

\section{Objectives}

To prospectively assess the accuracy of the Air-Smart Spirometer to measure forced expiratory volume in the first second $\left(\mathrm{FEV}_{1}\right)$ and forced vital capacity (FVC) in an SA population, and to investigate the ability of the device to reflect obstructive ventilatory impairment.

\section{Methods}

We conducted a prospective observational study at Tygerberg Hospital, Cape Town, SA, in which 200 consecutive patients and healthy volunteers were invited to participate. The study was approved by the Health Research Ethics Committee of Stellenbosch University (ref. no. S18/02/034). Participants were randomly (1:1) assigned to spirometry either with the mobile spirometer connected to a smartphone or the desktop spirometer first, followed by the other. Pulmonary function tests were performed in accordance with the 
current American Thoracic Society, European Respiratory Society and South African Thoracic Society guidelines. ${ }^{[7,13]}$ Desktop FEV Ind $_{1}$ and FVC were measured with a Jaeger Master Screen (Becton Dickinson, USA), which was calibrated daily, and mobile spirometry was done with the Air-Smart Spirometer (Pond Health Care Innovations, Sweden) (Fig. 1) connected to a smartphone following on-screen prompts in accordance with the manufacturer's specifications. ${ }^{[14]}$ The device has a turbine mechanism to perform measurements inside the disposable single-use nozzles. It does not require calibration, but parameters of age, sex and height are entered prior to spirometry. ${ }^{[14]}$ To perform spirometry, participants were asked to perform forced exhalation of air into the turbine. This air turns a motor, and the device registers the speed of the rotor and in turn transfers the data to the smartphone application. When the patient initiates exhalation, a chronometer switches on and changes its colour from red to green after 6 seconds of exhalation. ${ }^{[14]}$

Comorbidities and demographic data (sex, age, height, weight and self-reported ethnicity) of the participants were documented. We subsequently compared the $\mathrm{FEV}_{1} / \mathrm{FVC}$ ratio as well as the absolute $\mathrm{FEV}_{1}$ and FVC measurements, using each participant as their own control. Data analysis was performed using SPSS statistics, version 25 (IBM, USA). We considered a $p$-value $<0.05$ to be statistically significant. We did a Pearson correlation analysis to assess the strength of the linear relationship between the devices, but since correlation analysis does not detect bias, we conducted a BlandAltman analysis ${ }^{[15]}$ on all components of spirometry to assess the bias limits of agreement. We defined obstructive lung disease as an $\mathrm{FEV}_{1} / \mathrm{FVC}$ ratio $<0.7$ as measured by desktop spirometry in order to calculate the sensitivity, specificity, positive predictive value (PPV) and negative predictive value (NPV) of the Air-Smart Spirometer.

\section{Results}

Comorbidities in the 200 participants (mean (standard deviation, SD) age $42.2(21.3)$ years, $n=83$ males) included asthma $(n=74)$,

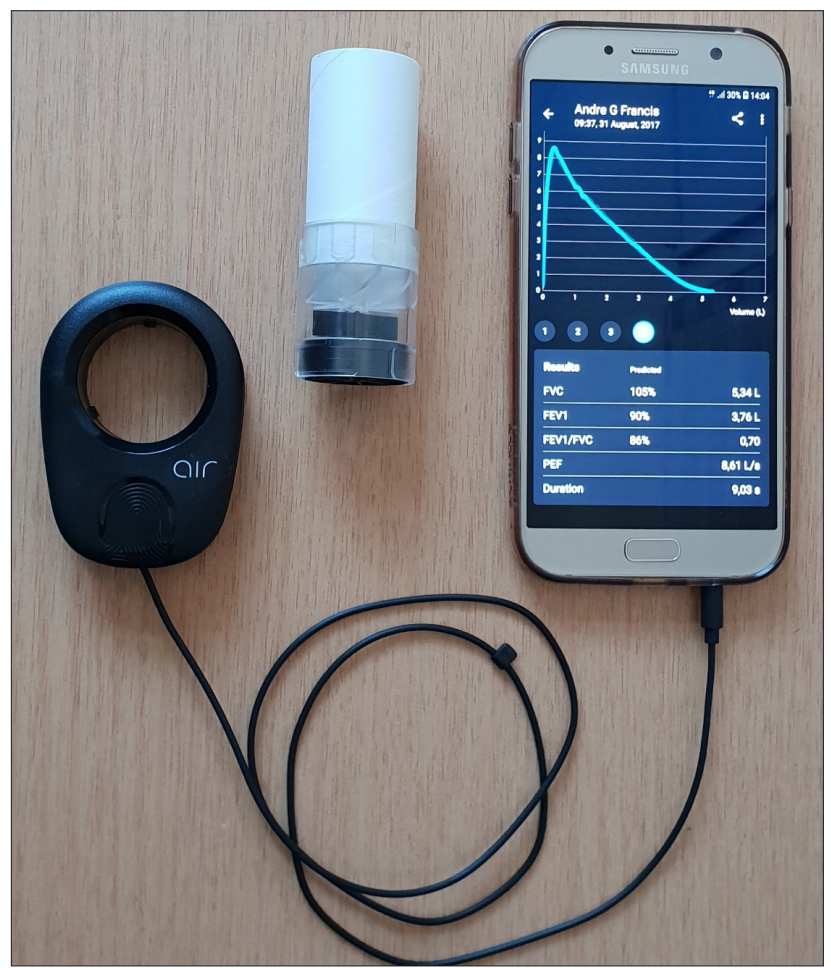

Fig. 1. The Air-Smart Spirometer (Pond Health Care Innovations, Sweden), connected to a standard smartphone.
COPD $(n=30)$, post-tuberculosis structural lung disease $(n=18)$, bronchiectasis $(n=14)$, lung masses $(n=12)$, sarcoidosis $(n=11)$, tuberculosis $(n=6)$, interstitial lung disease $(n=5)$, pulmonary hypertension $(n=3)$, pleural effusions $(n=3)$, pneumoconiosis $(n=3)$ and previous pneumonectomy $(n=2)$. Participants reported their race as mixed $(n=140)$, white $(n=41)$ and black African $(n=19)$.

There was a strong correlation between the $\mathrm{FEV}_{1}$ measured with the devices, with $r=0.951$. (Fig. 2). The mean bias between the two devices was very close to 0 and not statistically significantly different from 0 ( $p=0.969)$. The $95 \%$ limits of agreement for the difference in $\mathrm{FEV}_{1}$ on the Bland-Altman plot (Fig. 3) were $-35 \mathrm{~mL}$ to $+35 \mathrm{~mL}$. The plot shows a random distribution of the differences between the two devices with no specific pattern according to the mean of both devices and therefore no proportional bias present $(\beta=-0.018$, $p=0.252$ ).

For FVC we also observed a strong correlation, with $r=0.955$ (Fig. 4). The mean bias was $-0.128 \mathrm{~L}$, which was significantly different from $0(p<0.001)$. The Bland-Altman plot (Fig. 5) displayed a random distribution with the limits of agreement calculated at $-70 \mathrm{~mL}$ to $+45 \mathrm{~mL}$. There was a significant proportional bias ( $\beta=-0.045, p=0.039$ ), which can be observed in Fig. 5 as a regression line that has a slight negative slope indicating that as the mean of the two devices increased, the differences became more negative.

The $\mathrm{FEV}_{1} / \mathrm{FVC}$ ratio measured with the Air-Smart Spirometer showed a strong correlation with the ratios obtained from standard

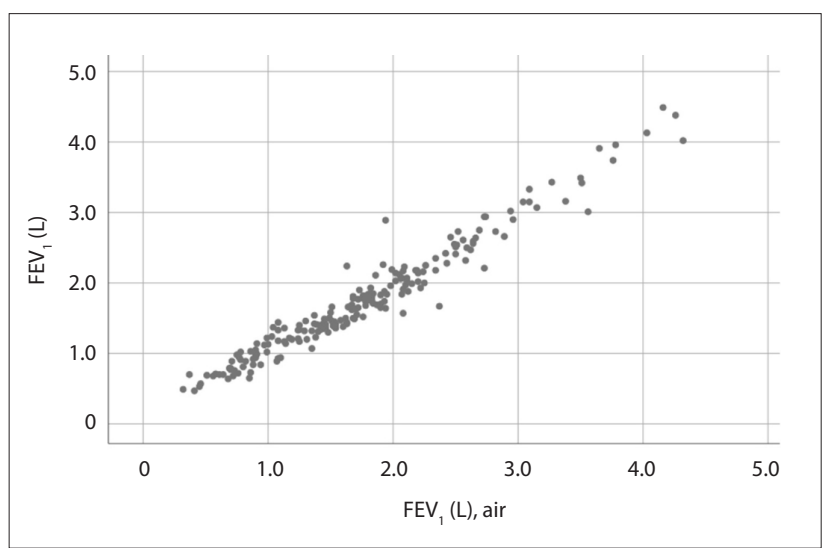

Fig. 2. Scatter plot of the FEV absolute values measured in the participants $(\mathrm{N}=200)$ with the Pearson correlation coefficient $(\mathrm{r}=0.951) .\left(F E V_{1}=\right.$ forced expiratory volume in the first second.)

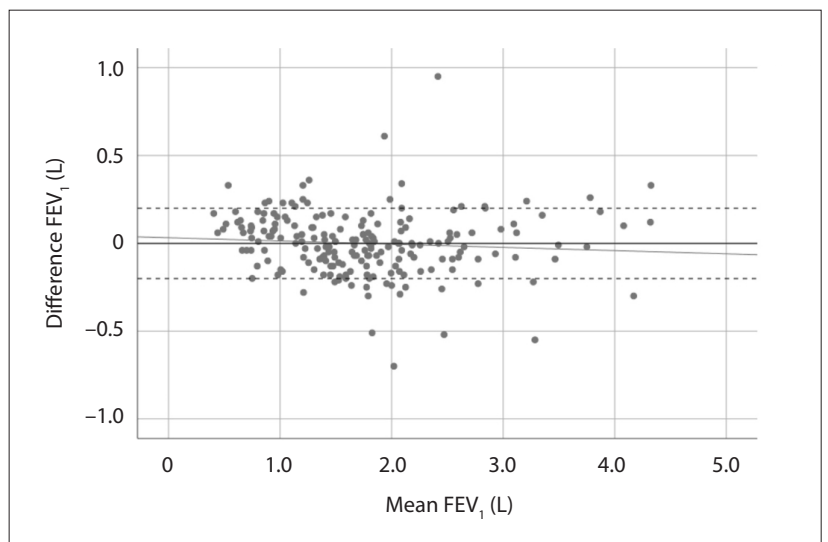

Fig. 3. Bland-Altman plot comparing the two measurement techniques for absolute $F E V_{1}$ values $(\mathrm{N}=200)(\mathrm{r}=0.007)$. $\left(F E V_{1}=\right.$ forced expiratory volume in the first second.) 


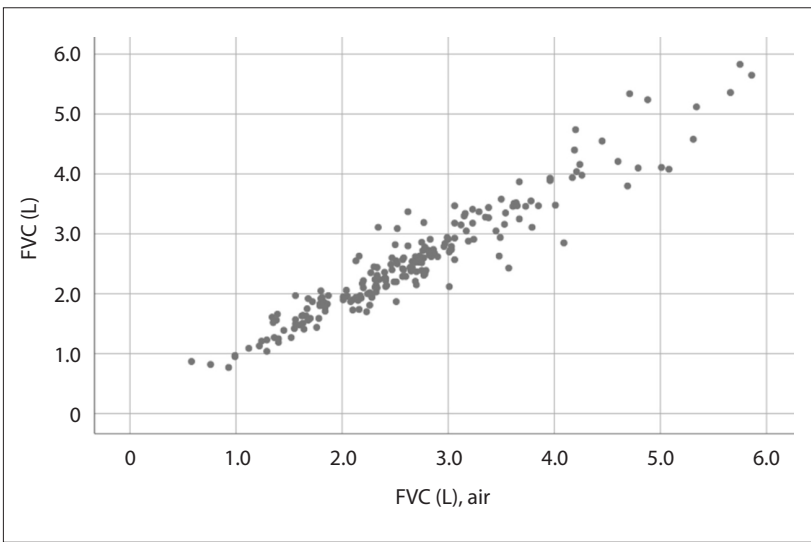

Fig. 4. Scatter plot of the FVC absolute values measured in the participants $(\mathrm{N}=200)$ with the Pearson correlation coefficient $(\mathrm{r}=0.955) .(F V C=$ forced vital capacity.)

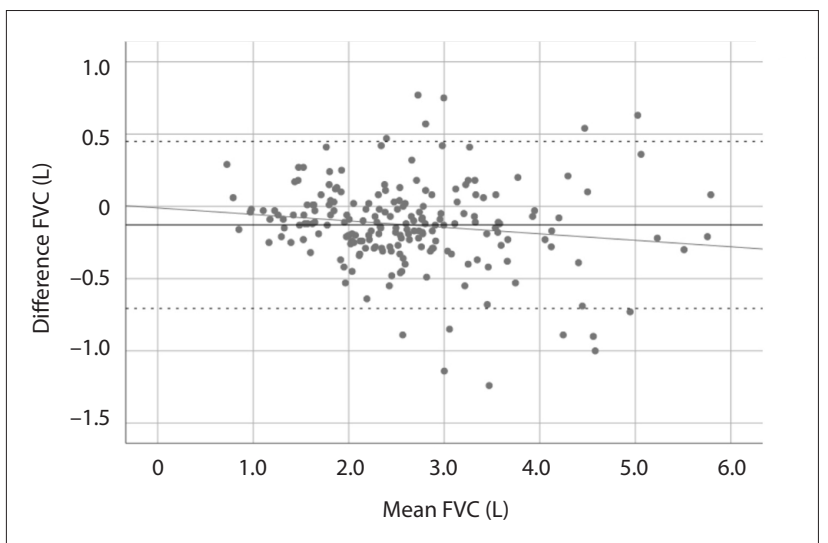

Fig. 5. Bland-Altman plot comparing the two measurement techniques for absolute FVC values $(\mathrm{N}=200)(\mathrm{r}=0.007)$. ( $F V C=$ forced vital capacity.)

spirometry, with $r=0.898$. The Air-Smart Spirometer had a sensitivity of $97.6 \%$ (95\% confidence interval (CI) 90.8 - 99.6), specificity of $74.4 \%$ (95\% CI $65.3-81.8$ ), PPV of $73.0 \%$ (95\% CI $63.6-80.8$ ) and NPV of $97.8 \%$ (95\% CI 91.4 - 99.6) for obstructive ventilatory impairment.

\section{Discussion}

We found a strong correlation between the absolute $\mathrm{FEV}_{1}$ and FVC values and the $\mathrm{FEV}_{1} / \mathrm{FVC}$ ratio measured with the Air-Smart Spirometer and conventional pulmonary function testing, with $r=0.951, r=0.955$ and $r=0.898$, respectively. Moreover, Bland-Altman analysis showed that while for $\mathrm{FEV}_{1}$ there was minimal bias, for FVC the bias was larger and statistically significantly different from 0 , but not clinically significant, as differences of $<100 \mathrm{~mL}$ are generally not considered significant. The Air-Smart Spirometer had a sensitivity of $97.6 \%$, specificity of $74.4 \%$, PPV of $73.0 \%$ and NPV of $97.8 \%$ for obstructive ventilatory impairment.

In a recent study by Ramos Hernández et al., ${ }^{[14]}$ the utility of the Air-Smart Spirometer device to detect obstructive ventilatory impairment was tested in 200 patients (mean (SD) age 57 (14) years). Obstruction was detected by conventional spirometry in 73 patients (40.1\%). Using an $\mathrm{FEV}_{1} / \mathrm{FVC}$ ratio $<0.7$ to detect obstruction with the Air-Smart Spirometer, the sensitivity was $90.4 \%$, specificity $97.2 \%$, PPV 95.7\% and NPV 93.7\% (compared with formal spirometry as the gold standard). The authors concluded that the device is simple, easy to use and accurate in detecting obstructive airway diseases. ${ }^{[14]}$
We observed a comparable sensitivity and NPV (making it an ideal screening test), but lower specificity and PPV.

We believe that our results have major implications for a resource-constrained healthcare system servicing a vast population, including rural areas. Many parts of Africa have poor accessibility to spirometers, and even when they are available, expertise in their proper maintenance, calibration, technical aspects and interpretation is limited. Mobile devices connected to smartphones or other devices may be a revolutionary advance in the early detection and screening of chronic pulmonary disorders in primary healthcare settings, and the onscreen prompts should enable nurses and other primary care providers to perform the tests with limited training. The linked smartphone also allows for data to be analysed off site, and despite some limitations may eventually aid in the better understanding of appropriate reference values in black African patients, which are currently lacking. ${ }^{[7,16]}$ Moreover, it may streamline referral to tertiary centres with limited capacity.

\section{Study limitations}

Our study has some limitations, including the facts that both sets of tests were performed by qualified pulmonary function technologists and that our study population included a relatively small number of black African patients. Future studies should focus on inexperienced personnel, and what the impact on accuracy (if any) may be should the mobile Air-Smart Spirometer be used in a primary healthcare setting.

\section{Conclusions}

The mobile Air-Smart Spirometer compared well with more conventional spirometry, making it an attractive and potentially affordable tool for screening purposes in a primary care setting. Moreover, it has a high sensitivity and NPV for obstructive ventilatory impairment.

\section{Declaration. None.}

Acknowledgements. We thank Sandoz South Africa for donating the AirSmart Spirometer.

Author contributions. CFNK and EdP initiated the study, analysed the data and wrote the manuscript. All authors assisted with the study design and data collection, and edited the manuscript. EMI critically reviewed the data analysis and edited the final draft of the manuscript.

Funding. None.

Conflicts of interest. None.

1. World Health Organization. The World Health Report 2002 - Reducing Risks, Promoting Healthy Life Geneva: WHO, 2002. http://www who int/whr/2002 (accessed 16 January 2010).

2. Oni T, Youngblood E, Boulle A, McGrath N, Wilkinson RJ, Levitt NS. Patterns of HIV, TB, and nonOni T, Youngblood E, Boulle A, McGrath N, Wilkinson RJ, Levitt NS. Patterns of HIV, TB, and non-
communicable disease multi-morbidity in peri-urban South Africa - a cross sectional study. BMC communicable disease multi-morbidity in peri-urban South Africa

3. Furlow B. South Africa confronts colliding respiratory epidemics. Lancet Respir Med 2014;2(11):874875. https://doi.org/10.1016/S2213-2600(14)70241-3

4. Van Zyl Smit RN, Pai M, Yew WW, et al. Global lung health: The colliding respiratory epidemics of tuberculosis, tobacco smoking, HIV and COPD. Eur Respir J 2010;35(1):27-33. https://doi. org/10.1183/09031936.00072909

5. Vogelmeier CF, Criner GJ, Martinez FJ, et al. Global Strategy for the Diagnosis, Management, and Prevention of Chronic Obstructive Lung Disease 2017 Report: GOLD Executive Summary. Am Respir Crit Care Med 2017;195(5):557-582. https://doi.org/10.1164/rccm.201701-0218PP

6. Becker AB, Abrams EM. Asthma guidelines: The Global Initiative for Asthma in relation to national guidelines. Curr Opin Allergy Clin Immunol 2017;17(2):99-103. https://doi.org/10.1097/ ACI.0000000000000346

7. Koegelenberg CF, Swart F, Irusen EM. Guideline for office spirometry in adults, 2012. S Afr Med J 2013;103(1):52-62. https:///doi.org/10.7196/SAMI.6197

8. Duong-Quy S, Hua-Huy T, Mai-Huu-Thanh B, Doan-Thi-Quynh N, Le-Quang K, Nguyen-Van H. Early detection of smoking related chronic obstructive pulmonary disease in Vietnam. Rev Mal Respir Early detection of smoking related chronic obstructive pulmo
2009;26(3):267-274. https://doi.org/S0761-8425(09)72583-5

9. Kaufmann M, Hartl S, Geyer K, Breyer MK, Burghuber OC. Measuring $\mathrm{FEV}_{1}$ for detecting early airway obstruction in the primary care setting: Quality and utility of the new PiKo-6 device. Respiration 2009;78:161-167. https://doi.org/10.1159/000197466 
10. Represas-Represas C, Leiro-Fernández V, Mallo-Alonso R, Botana-Rial MI, Tilve-Gómez A, FernándezVillar A. Excessive dynamic airway collapse in a small cohort of chronic obstructive pulmonary disease patients. Ann Thorac Med 2015;10(2):118-122. https://doi.org/10.4103/1817-1737.150733

11. Represas-Represas C, Fernandez-Villar A, Ruano-Raviña A, Priegue-Carrera A, Botana-Rial M. Screening for chronic obstructive pulmonary disease: Validity and reliability of a portable device in Screening for chronic obstructive pulmonary disease: Validity and reliability of a portable device in
non-specialized healthcare settings. PLoS One 2016;11(1):e0145571. https://doi.org/10.1371/journal.

12. Graham-Barr R, Stemple KJ, Mesia-Vela S, et al. Reproducibility and validity of a handheld spirometer Respir Care 2008;53(4):433-441.

13. Quanjer PH, Stanojevic S, Cole TJ, et al. Multi-ethnic reference values for spirometry for the $3-95$ year age range: The Global Lung Function 2012 equations. Eur Respir J 2012;40:1324-1343. https://doi. org $/ 10.1183 / 09031936.00080312$
14. Ramos Hernández C, Núñez Fernández M, Pallares Sanmartín A, et al. Validation of the portable Air-Smart Spirometer. PLoS One 2018:13(2):e0192789. https://doi.org/10.1371/journal.pone.0192789 15. Giavarina D. Understanding Bland Altman analysis. Biochem Med 2015;25(2):141-151. https://doi,
. org/10.11613/BM.2015.015

16. Koegelenberg CF, Swart F, Irusen EM. Prediction equations for spirometry in South Africa. S Afr Med J 2013;103(9):597. https://doi.org/10.7196/SAMJ.7298

Accepted 21 February 2019. 